\title{
Factores determinantes en la expresión del sujeto pronominal en el corpus PRESEEA de Granada
}

\author{
Antonio Manjón-Cabeza Cruz ${ }^{1}$ \\ Francisca Pose Furest \\ Francisco José Sánchez García \\ Universidad de Granada
}

\begin{abstract}
Resumen
El objetivo de este trabajo es examinar la relación existente entre determinadas variables y la mayor o menor presencia / ausencia del pronombre personal sujeto junto a un verbo en forma personal en el español hablado de Granada (España). Con ello, pretendemos poder establecer qué factores intervienen en el empleo del sujeto pronominal frente al cero fonético en determinados enunciados. Para precisar los factores condicionantes se ha seguido la guía de codificación propuesta por Bentivoglio, Ortiz y Silva-Corvalán (2011) para el proyecto PRESEEA.

Tras los tratamientos estadísticos estándares, comprobamos, en primer lugar, que la tasa de aparición del pronombre personal es de las más bajas del mundo hispánico; y, en segundo lugar, que son significativas las siguientes variables: persona gramatical, especificidad, ambigüedad de la forma verbal, clase semántica del
\end{abstract}

\footnotetext{
1 Para correspondencia, dirigirse a: Antonio Manjón-Cabeza Cruz (amanjoncabeza@ ugr.es), Departamento de Lengua Española, Facultad de Filosofía y Letras, Universidad de Granada, E - 18071 GRANADA, España.
} 
verbo, forma perifrástica, correferencialidad, comienzo de turno de habla y edad. El artículo se centrará en el comentario y explicación de las posibles interrelaciones de los valores obtenidos.

Palabras clave: sujeto pronominal, PRESEEA, Granada, factores significativos.

\title{
DETERMINANT FACTORS IN THE EXPRESSION OF THE PRONOMINAL SUBJECT IN THE PRESEEA CORPUS OF GRANADA
}

\begin{abstract}
The objective of this study is to examine the relationship between certain variables and the presence/absence to a greater or lesser degree of the personal pronoun together with a conjugated verb in spoken Spanish of Granada (Spain). By doing so, we want to determine the factors involved in using the pronominal subject instead of the phonetic zero in certain statements. In order to define the determining factors, we have followed the coding guidelines proposed for the PRESEEA Project by Bentivoglio, Ortiz and Silva-Corvalán (2011). After the standard statistical processing firstly we verified that the prevalence rate of the personal pronoun is one of the lowest in the Spanish-speaking world and secondly, that the following variables are significant: grammatical person, specificity, ambiguity of the verbal form, semantic class of the verb, periphrastic form, co-reference, the beginning of speech turn and age. This paper focuses on the comments and explanations regarding the possible interrelationships among the values obtained.
\end{abstract}

Keywords: pronominal subject, PRESEEA, Granada, significant factors.

Recibido: 28/03/2016

Aceptado: 04/07/2016 


\section{INTRODUCCIÓN}

Este trabajo ${ }^{2}$ trata de los factores que explican la aparición o ausencia del pronombre personal sujeto. Es conocido que el español es una lengua pronoun-dropping (pro-drop), que permite la ausencia del sujeto pronominal. Así lo comprobamos en (1):

(1) Pues mira yo// salgo por la mañana/// (Ø) cojo mi coche que (Ø) ya me he espabila[d]o un poquillo sí yo ya conduzco/ (Ø) ya soy una mujer/ [GRAN-M23-10] ,

en el que observamos cinco verbos en primera persona y solo dos pronombres explícitos.

El principal objetivo de este estudio estriba en contribuir al conocimiento de los condicionamientos sociolingüísticos de la aparición del pronombre personal sujeto siguiendo las pautas de un proyecto panhispánico (PRESEEA: Proyecto para el estudio sociolingüístico del español de España y América) y de una guía de codificación unificada que permita comparar los resultados obtenidos en Granada (España) con los de otras comunidades de habla hispánica.

La comunidad granadina presenta unas particularidades lingüísticas que la hacen especialmente interesante como objeto de estudio de un hecho de variación gramatical ampliamente tratado en la sociolingüística del español. No podemos dejar de citar que se trata de una comunidad donde se está dando un proceso de convergencia con el estándar del español europeo y una consiguiente estigmatización de la mayoría de las variantes vernáculas.

Uno de los fenómenos fónicos vernáculos más caracterizadores de la comunidad es la proyección o abertura vocálica, que parece escapar a la creciente estandarización y que es, según creemos, una característica que se puede relacionar con la presencia o ausencia de los sujetos pronominales.

2 Nuestro estudio se ha realizado en el marco del Proyecto PASOS-Granada (Patrones sociolingüísticos del español de Granada), financiado por el Ministerio de Ciencia e Innovación (Ref. FF12011-29189 - C05-05) y ECOPASOS-Granada financiado por MINECO/FEDER (Ref. FFI2015-68171-C5-2-P), integrados en el proyecto PRESEEA (Proyecto para el estudio sociolingüístico del español de España y América).

3 El sistema de codificación de los informantes puede consultarse en Moya Corral (2009: 25). Para esta codificación se han seguido las pautas indicadas por el PRESEEA, que deben ser comunes a todos los equipos. Así, GRAN (ciudad de Granada), M (Mujer), 2 (Generación $2^{\mathrm{a}}$ : entre 35 y 54 años), 3 (Instrucción 3, nivel superior) y 10 (número de orden, informante 10). Asimismo, hemos preferido mantener las barras /, //, /// para marcar las pausas. 
Este estudio, por tanto, debe servir para responder a varias hipótesis sobre la presencia de pronombres personales en función de sujeto en esta comunidad del sur de España: ¿Los hablantes granadinos se alinean con aquellos que mantienen la /-s/ final o con los que tienen tendencia a la pérdida? ¿Los resultados de Granada convergen con los de otras comunidades de habla o muestran diferencias significativas? ¿En qué medida contribuyen a la pérdida o presencia del sujeto pronominal los distintos factores gramaticales, fonéticos, semánticos, pragmático-textuales y sociales? A responder a estas preguntas están dedicadas las páginas que siguen.

\section{MARCO TEÓRICO}

La tradición contempló generalmente el uso del pronombre personal sujeto como algo meramente enfático. De este modo, la alternancia se consideró una variante libre cuya presencia no debía alterar el significado del enunciado. Para la RAE, la expresión del pronombre personal sujeto iba ligada a aquellos casos en los que el hablante necesitaba determinar más el sujeto: la ambigüedad y el énfasis (Esbozo 1973: 421). Sin embargo, en la Nueva Gramática (2009: 2555 y ss.) se contempla el uso de este pronombre como un recurso capaz de desempeñar una función informativa, la focal (2009: 2555). Aquí se advierte también de dos aspectos que influyen en la presencia del pronombre personal sujeto: uno es de naturaleza morfológica y se pone de ejemplo la primera y tercera persona del pretérito imperfecto de indicativo; otro es de naturaleza léxica. En este caso, el ejemplo ofrecido es el pronombre usted, que formado con los imperativos no posee carácter contrastivo: "Siéntese usted, por favor".

Partimos, pues, de la base de que la presencia del pronombre personal sujeto no es aleatoria en español, sino que, como ya se intuía, responde a diversos factores. La cuestión gira ahora en torno a la determinación de qué factores concretos originan su uso.

Entre las causas que favorecen la presencia del pronombre personal sujeto en español, los estudios coinciden en señalar las siguientes:

- La intención de contraste, centrada en destacar la actitud del hablante frente al otro. La función pragmática de la presencia del sujeto pronominal se observa, entre otros aspectos, en el fenómeno de la alo-repetición, entendiendo como tal un hecho que provoca a menudo la reproducción 
por parte del interlocutor del pronombre sujeto. O la correferencialidad que, según Silva-Corvalán (1982), influye en la mayor o menor presencia del pronombre personal sujeto.

- La semántica verbal. Verbos con un marcado carácter subjetivo determinan la presencia del sujeto pronominal. Los verbos más proclives a llevar de forma explícita el pronombre personal sujeto son aquellos que pertenecen al campo semántico del conocimiento, percepción intelectual o los que expresan opinión (Enríquez 1984).

- La ambigüedad, originada por razones morfofonológicas. No solo causada por la coincidencia de la primera persona del singular del pretérito imperfecto de indicativo y subjuntivo y del condicional (SilvaCorvalán 1982) con las terceras personas de los mismos tiempos, sino también por la pérdida de la /-s/ en determinadas variedades del español que provoca la concurrencia de la primera y segunda persona (Hochberg 1986).

La bibliografía ha tratado en numerosas ocasiones este asunto, si bien con diferentes enfoques y, lo más importante, con diversos y no siempre adecuados corpora. Podemos citar trabajos como los de Barrenechea y Alonso (1977), Bentivoglio (1980), Silva-Corvalán (1982) o Enríquez (1984). Y algunos más actuales como los de Orozco y Guy (2008), Erker y Guy (2012), Shin y Otheguy (2013), el reciente volumen recopilatorio de Carvalho, Orozco y Shin (2015), la nueva aportación de Silva-Corvalán (2015) o la monografía de Peškova (2015), en los que podemos comprobar la diversidad de enfoques y corpora a los que aludíamos más arriba.

La posibilidad de alternancia del pronombre sujeto en español, más que responder a una cuestión estilística, que no modifica el contenido de lo dicho, obedece a condicionamientos léxico-pragmático-morfofonológicos. En palabras de Silva-Corvalán (2003: 850): «la expresión llamada "opcional" del sujeto parece válida en un número cada vez más limitado de contextos discursivos». La autora demuestra de forma práctica en qué casos es posible prescindir de la presencia del sujeto pronominal y en cuáles su expresión resulta obligatoria. Así, factores como la correferencialidad con el sujeto previo, la topicalidad y la ambigüedad de la forma verbal no exigen la expresión del sujeto pronominal. Ahora bien, cuando es preciso aclarar el referente o cuando se trata de un sujeto focal (información nueva o contrastiva) la expresión es obligatoria (vid. Silva-Corvalán 2003: 850). 


\section{ANÁLISIS DE LOS DATOS}

Para analizar los factores que influyen en la presencia o ausencia del sujeto pronominal, nos basamos en el corpus PRESEEA (Proyecto para el estudio sociolingüístico del español de España y América) de Granada (España), publicado en Moya Corral y otros (2007, 2008 y 2009). Es un corpus oral, obtenido en función de un muestreo por cuotas de afijación uniforme. Las tres variables sociales son: sexo, edad y nivel de instrucción. La distribución de los hablantes queda reflejada en la Tabla I:

Tabla 1: Distribución de los hablantes del corpus PRESEEA (Granada)

\begin{tabular}{|c|c|c|c|c|c|c|c|}
\hline \multirow{2}{*}{54 informantes } & \multicolumn{6}{|c|}{ Nivel de estudios } \\
\cline { 3 - 9 } \multicolumn{2}{|c|}{} & \multicolumn{2}{|c|}{ Primarios } & \multicolumn{2}{c|}{ Secundarios } & \multicolumn{2}{c|}{ Universitarios } \\
\cline { 2 - 8 } & Mujer & Hombre & Mujer & Hombre & Mujer & Hombre \\
\hline \multirow{3}{*}{ Edad } & $>19 \mathrm{y}<34$ & 3 & 3 & 3 & 3 & 3 & 3 \\
\cline { 2 - 8 } & $>34 \mathrm{y}>54$ & 3 & 3 & 3 & 3 & 3 & 3 \\
\cline { 2 - 8 } & $\geq 55$ & 3 & 3 & 3 & 3 & 3 & 3 \\
\hline
\end{tabular}

De cada hablante se seleccionaron cinco minutos, los comprendidos entre el diez y el quince. Se procedió a la anotación de aquellos casos en que era posible la alternancia, como los que aparecen en (2):

(2) El Corpus muy bien// y cuando (Ø) me casé/ mejor todavía/ (Ø) iba a los toros (Ø) iba/ pues/ a todos los sitios/ y (Ø) me... lo pasaba muy bien/ yo aquí lo he pasado muy bien// con mi marido (Ø) lo he pasado muy bien [GRAN-M31-052].

No se anotaron los casos donde no es posible la alternancia, bien por la presencia necesaria del sujeto, como en los numerosos casos de yo qué sé / qué sé yo, que podemos ejemplificar con (3):

(3) Y también pues te/ estabas dormido y escuchabas la banda y en seguida te despertabas/// yo qué sé. [GRAN-H11-38],

bien por su ausencia obligada, como en (4) donde hay correferencia en la proposición de relativo y no es posible la presencia de ellos:

(4) Hay un chiste muy bueno/ es una pareja de ancianos// que se acercan por una farmacia [GRAN-M33-17]. 
Obtuvimos así 3415 ejemplos, con un porcentaje de aparición del pronombre personal de $17,50 \%$. Es una tasa que podemos considerar, sin duda, muy reducida si la comparamos con las obtenidas para otros corpus orales (datos resumidos en Lastra y Martín Butragueño 2015). Solo Peškova (2015: 141) ofrece un porcentaje menor $(17,00 \%)$, aunque hay que recordar que su corpus no es comparable porque los hablantes son de un grupo "bastante homogéneo" y "los aspectos sociales no han sido tomados en consideración" (Peškova 2015: 216).

Los ejemplos han sido codificados según la guía propuesta por Bentivoglio, Ortiz y Silva-Corvalán (2011). En esta codificación se contempla una variable dependiente, la expresión o no del sujeto pronominal; y quince independientes, de las que cuatro son sociales: edad, sexo, nivel educativo y variedad geográfica; y once son lingüísticas: persona del sujeto, especificidad del mismo, modo, tiempo verbal, progresividad, perfectividad, ambigüedad de la forma verbal, clase semántica del verbo, tipo de cláusula, correferencialidad y turno de habla.

Esta guía común de codificación ha sido modificada parcialmente, sobre todo para añadir otras variables independientes e intentar así afinar los resultados. Las nuevas variables independientes son:

- Forma perifrástica, donde se consideran las variantes perífrasis modal (puede cantar), aspectual (está cantando) mixta (puede estar cantando) $\mathrm{y}$ sin forma perifrástica.

- Hiperónimo verbal (ser, estar, ir, hacer, decir, saber, tener y dar, frente al resto de los verbos $\left.{ }^{4}\right)$.

- Estilo (narrativo, argumentativo+explicativo, descriptivo, diálogo rápido y otros).

- Valencia verbal (cerovalentes, monovalentes, divalentes o trivalentes ${ }^{5}$ ).

- Pronominalidad (verbos pronominales y no pronominales).

\footnotetext{
4 Aunque la nómina de hiperónimos - palabras con significado muy general que pueden sustituir en el discurso a otras de significado más específico- se puede ampliar, elegimos esta lista como representativa de hiperónimos de verbos de estado (ser y estar), movimiento (ir), actividades (hacer), dicendi (decir), procesos mentales (saber), posesión (tener) y transferencia de posesión (dar), que son grupos semánticos tradicionalmente usados en la descripción lingüística hispánica.

5 Los discutidos y, por otra parte, escasísimos casos de verbos tetravalentes se han agrupado con los trivalentes.
} 
Tras el análisis de diferencias de medias (ANOVA) con el programa de tratamiento estadístico IBM SPSS 20, hallamos que ocho variables independientes resultan significativas. Así, como se refleja en la Tabla 2 , observamos que obtienen una significación asintótica o $\mathrm{p}$ valor $<0.05$ las siguientes variables ${ }^{6}$ : persona gramatical, especificidad, ambigüedad de la forma verbal, clase semántica del verbo, forma perifrástica, correferencialidad, comienzo de turno de habla y edad.

Tabla 2. Análisis de diferencias de medias (ANOVA) para todas las variables

\begin{tabular}{|l|c|c|l|c|c|}
\hline Variable & $\mathrm{F}$ & Sig. & Variable & $\mathrm{F}$ & Sig. \\
\hline Persona & 145.870 & .000 & Hiperónimo & .035 & .851 \\
\hline Especificidad & 61.393 & .000 & tipo modal & 2.677 & .102 \\
\hline Modo & 1.267 & .260 & Correferencia & 19.365 & .000 \\
\hline Tiempo & 3.347 & .067 & Turno & 7.146 & .008 \\
\hline $\begin{array}{l}\text { progresividad } \\
\text { (aspecto) }\end{array}$ & .543 & .461 & Estilo & 3.491 & .062 \\
\hline Perfectividad & 2.395 & .122 & Edad & 18.460 & .000 \\
\hline Ambigüedad & 23.866 & .000 & Sexo & .554 & .457 \\
\hline clase semántica & 25.614 & .000 & Nivel & 1.193 & .275 \\
\hline $\begin{array}{l}\text { semántica } \\
\text { aspectual }\end{array}$ & 2.957 & .086 & Valencia & .133 & .715 \\
\hline Perífrasis & 9.748 & .002 & Pronominal & 1.013 & .314 \\
\hline
\end{tabular}

Estos resultados confirman que la ausencia o presencia del sujeto pronominal en español está influida por variados factores que interrelacionan. Asimismo, nos permiten eliminar en posteriores tratamientos estadísticos aquellas variables con un $\mathrm{p}$ valor $>0,05$.

A continuación realizamos un análisis de regresión logística de efectos fijos, para el que utilizamos el programa estadístico Goldvarb X, que sirve para medir los pesos probabilísticos de aquellos factores seleccionados como significativos para explicar la variabilidad, así como su fuerza de factor (Sankoff, Tagliamonte y Smith 2005). Los resultados aplicados a las ocho variables anteriores aparecen en la tabla 3.

\footnotetext{
6 Hay algunas variables que no alcanzan el p-valor $<0.05$, pero que están cerca. Es el caso del tiempo verbal, la semántica aspectual y el estilo. Creemos que deberán incluirse en otros estudios para comprobar su significatividad en otros corpora.
} 
Tabla 3. Análisis de regresión logística de efectos fijos ${ }^{7}$

\begin{tabular}{|l|l|l|l|l|l|}
\hline Factor & $\begin{array}{l}\text { Peso } \\
\text { probabilístico }\end{array}$ & p-valor & $\begin{array}{l}\text { Fuerza } \\
\text { de factor }\end{array}$ & $\%$ & $\mathrm{n} /$ total \\
\hline Persona & & 0.000 & 446 & & \\
\hline $1 \mathrm{~s}$ & 0.646 & & & 24.7 & $424 / 1715$ \\
\hline $2 \mathrm{~s}$ & 0.496 & & & 15.0 & $68 / 452$ \\
\hline $3 \mathrm{~s}$ & 0.516 & & & 16.1 & $64 / 397$ \\
\hline $1 \mathrm{p}$ & 0.254 & & & 5.8 & $25 / 432$ \\
\hline $3 \mathrm{p}$ & 0.200 & & & 4.3 & $18 / 419$ \\
\hline Especificidad & & 0,000 & 289 & & \\
\hline Específico & 0.555 & & & 19.9 & $558 / 2804$ \\
\hline No específico & 0.266 & & & 6.7 & $41 / 611$ \\
\hline Ambigüedad & & 0.000 & 234 & & \\
\hline Ambiguo & 0.701 & & & 32.6 & $118 / 362$ \\
\hline No ambiguo & 0.467 & & & 15.3 & $429 / 2808$ \\
\hline Parcial & 0.566 & & & 21.2 & $52 / 245$ \\
\hline Semántica & & 0.000 & 209 & & \\
\hline Mental & 0.648 & & & 27.6 & $163 / 591$ \\
\hline Estado & 0.514 & & & 17.3 & $133 / 741$ \\
\hline Dicendi & 0.476 & & & 15.8 & $41 / 260$ \\
\hline Actividad & 0.439 & & & 13.9 & $193 / 1391$ \\
\hline Aspectual & 0.487 & & & 16.4 & $10 / 61$ \\
\hline
\end{tabular}

7 Conviene aclarar tres datos específicos de la tabla 3 antes de pasar a la discusión de los resultados obtenidos. El primer dato tiene que ver con la variable "Perífrasis". Puesto que la variante "perífrasis mixta" solo ha ofrecido diez casos en nuestro corpus, hemos efectuado su recodificación en los otros dos tipos de perífrasis, según correspondiera, teniendo en cuenta la parte final de la misma. De este modo, la perífrasis mixta que aparece en Bueno / pues mucho me temo que le voy a tener que subir // dos euros [GRAN-M33-17] ha sido recodificada en el grupo de las perífrasis modales (tener que subir) y no en el de las aspectuales (voy a tener). El segundo dato se refiere a la variable "Correferencia", ya que solo la hemos cuantificado dentro del mismo turno de habla, no cuando el informante comenzaba turno después de una intervención de la entrevistadora. El tercer dato resaltable es la inexistencia de la segunda persona del plural, consecuencia lógica, derivada del registro empleado en la obtención del corpus que analizamos: la entrevista semidirigida. En estas entrevistas han intervenido el entrevistador y el informante, por tanto, la presencia del pronombre vosotros es casi inexistente. 


\begin{tabular}{|l|l|l|l|l|l|}
\hline Movimiento & 0.478 & & & 15.9 & $59 / 371$ \\
\hline Perífrasis & & 0.006 & 148 & & \\
\hline No & 0.515 & & & 18.3 & $544 / 2976$ \\
\hline Modal & 0.467 & & & 15.6 & $24 / 154$ \\
\hline Aspectual & 0.367 & & & 10.9 & $31 / 285$ \\
\hline Edad & & 0.000 & 131 & & \\
\hline Jóvenes & 0.419 & & & 13.0 & $159 / 1219$ \\
\hline Adultos & 0.550 & & & 20.3 & $238 / 1171$ \\
\hline Mayores & 0.540 & & & 19.7 & $202 / 1025$ \\
\hline Correferencia & & 0.000 & 121 & & \\
\hline Corr. con suj. & 0.439 & & & 14.1 & $174 / 1235$ \\
\hline Corr. Otro & 0.560 & & & 21.1 & $27 / 128$ \\
\hline No correfer. & 0.520 & & & 18.5 & $337 / 1820$ \\
\hline Turno & & 0.010 & 90 & & \\
\hline Mismo & 0.491 & & & 17.0 & $520 / 3067$ \\
\hline Cambio & 0.581 & & & 22.7 & $79 / 348$ \\
\hline
\end{tabular}

Este análisis selecciona las variables que favorecen la presencia del pronombre sujeto y se presentan ordenadas según la fuerza del factor, que se obtiene comparando los respectivos rangos que resultan de la diferencia entre las probabilidades mayor y menor de cada grupo de factores (Hernández Campoy y Almeida 2005: 268-269). En la segunda columna se recoge el peso probabilístico de cada factor: se entiende que se favorece el valor de aplicación si es mayor que 0.50 y lo desfavorece si es menor de 0.50 . Asimismo, los resultados muestran que el input general es 0.175 y el $L o g$ likelihood o Logaritmo de verosimilitud es -1585.756.

\section{DISCUSIÓN}

\subsection{Persona}

Al igual que sucede en todos los estudios previos, la persona gramatical del verbo es determinante para la tendencia a la selección o al rechazo del sujeto pronominal. Como se observa en la Tabla 3, la primera persona es 
la más proclive a aparecer, mientras que los pronombre personales plurales tienden a no ser explicitados. Parece claro que el egocentrismo humano se refleja en la lengua (Givon 1976); además, las entrevistas semidirigidas condicionan la aparición de unos determinados pronombres, el yo y el tú, como los pronombres del diálogo que son (Enríquez 1984: 191). En palabras de Claes (2011: 200) "el comportamiento asociado a la persona y el número gramatical no constituye una característica de la gramática de los dialectos bajo análisis, sino del diasistema hispánico".

De todas maneras, como las variables interrelacionan, no podemos perder de vista que la mayor o menor presencia de los distintos pronombres personales está influida por otros factores. En Manjón, Pose y Sánchez (en prensa) hemos analizado cómo influyen en la aparición del sujeto pronominal -y cómo se interrelacionan- el factor social edad, la persona del sujeto y su especificidad. Por este motivo no ahondaremos aquí en las interrelaciones halladas en esos tres factores. Asimismo, tampoco se debe olvidar que las formas verbales ambiguas -que, como veremos, promueven la aparición del pronombre personal sujeto-, son las del singular, concretamente la primera y la tercera, hecho que puede también contribuir a que sean las personas más explicitadas.

\subsection{ESPECIFICIDAD}

A la vista de los resultados, es evidente que expresiones inespecíficas como las de los verbos hacian y repartían en (5) tienen una clara tendencia al rechazo del pronombre personal, ya que muestran un peso probabilístico de 0,266-ver Tabla 3-:

(5) Pues luego después que ya (Ø) hacían la procesión y todo eso// pues los roscos (Ø) los repartían gratuitos a todo el que quisiera ir. [GRAN-M33-016]

Además, no podemos perder de vista que en numerosas ocasiones esa inespecificidad hace que se emplee el cuantificador uno/a en sustitución de cualquier persona ${ }^{8}$.

En Manjón, Pose y Sánchez (en prensa) demostramos que, en Granada, las generaciones más jóvenes son las que promueven la inespecificidad

\footnotetext{
8 En Manjón, Pose y Sánchez (en prensa) aducimos varias razones que nos llevan a estudiar la presencia de los cuantificadores sujetos de manera separada a la de los pronombres personales.
} 
en el discurso, como un modo de refuerzo de la solidaridad y familiaridad (Brown y Gillman 1960).

\subsection{AMBIGÜEDAD}

La ambigüedad de la forma verbal es un factor que influye en la presencia del pronombre personal sujeto. Así lo han constatado tanto Silva-Corvalán (1982) en su estudio del español de Estados Unidos, como Bentivoglio (1980 y 1987) para el español de Venezuela, entre otros estudios.

Llegados a este punto, conviene insistir en la doble vertiente que presenta el factor ambigüedad: la ambigüedad de formas verbales estándares, caso de las primeras y terceras personas de los condicionales y de los pretéritos imperfectos y pluscuamperfectos tanto de indicativo, como de subjuntivo, factor aducido por Silva-Corvalán (1982); y la ambigüedad derivada de la pérdida de la $/-\mathrm{s} /$ en numerosas variedades del español, que tiende a igualar segundas y terceras personas: tú canta(s), ella canta. Este último tipo de ambigüedad ha dado pie a la conocida como hipótesis funcional (Hochberg 1986), según la cual la presencia del sujeto pronominal será mayor en aquellas variedades del español donde se produce esa pérdida, ya que contribuye a la desambiguación.

Esta hipótesis hay que tomarla con cautela, al menos en el caso de Granada. Es cierto que en esta ciudad está generalizada la pérdida de $/-\mathrm{s} /$. Según Tejada (2015: 83), en posición final, la elisión de la /-s/ alcanza un rotundo 94,6\%, seguida de la aspiración, con 4,6\%. El resto está constituido por variantes muy minoritarias. Podríamos, por tanto, cometer el error de tratar nuestros datos comparándolos con los de las variedades donde la presencia del pronombre se relaciona con la pérdida de /-s/. Pero no se debe olvidar que también es norma en Granada la abertura o proyección vocálica de las vocales finales de sílaba o palabra. Por ello, podemos afirmar que no se produce ambigüedad de formas entre la segunda y tercera persona, lo que asemeja en el funcionamiento gramatical, tal como señala Moya Corral (2013), la variedad granadina a las variedades caracterizadas por el mantenimiento de la /-s/, como Madrid o México DF.

9 Es un hecho que ha sido inexplicablemente obviado en otros estudios, como en Ranson (1991), para Puente Genil (Córdoba, España), donde las vocales proyectadas también están generalizadas. 
Por esta razón, en el caso de Granada, solo se puede estudiar el posible papel desambiguador del pronombre en las mismas formas verbales que en el español de Madrid o de México DF, es decir, las primeras y terceras personas del singular de algunos tiempos verbales, como los pretéritos imperfectos de indicativo que presentamos en (6), en el que se comprueba la presencia de los sujetos pronominales con formas verbales equívocas:

(6) Pero/ ahora está/ un poquito agrandado porque compraron el $\mathrm{piso} / \mathrm{mm}$ de... del vecino// y lo agrandaron// y yo tenía dos pisos// yo tenía/ bueno el/ el local// y/ y otro piso donde yo vivía// y donde en fin/ los bajos de mi dormitorio y eso// que lo agrandé// [GRAN-H32-033].

Si se observa la Tabla 3, se confirma la hipótesis de que las formas verbales ambiguas favorecen el empleo del pronombre sujeto con mayor frecuencia que cuando se utilizan formas verbales no ambiguas. De hecho, el peso probabilístico más alto de toda la tabla es el que aparece con las formas ambiguas $(0,701)$.

No obstante, la rotundidad de estos datos hay que matizarla, porque no debemos olvidar las palabras de Silva-Corvalán (2001: 169) cuando señalaba: "Los factores que parecen determinar la realización fonética variable de los pronombres sujeto en español oral son complejísimos". No olvidemos en este punto que las formas ambiguas son casi exclusivamente las de primera y tercera persona del singular, precisamente las favorecedoras de la expresión del sujeto, frente a las otras formas, especialmente las del plural.

A pesar de estas salvedades, creemos que la ambigüedad es un factor importante para explicar la presencia del sujeto pronominal en nuestro corpus. Podemos llegar a esa conclusión de dos maneras. La primera consiste en comparar los porcentajes de presencia del pronombre solo para aquellas formas que pueden ser ambiguas, es decir, las personas primera y tercera, como se muestra en el Gráfico 1. 


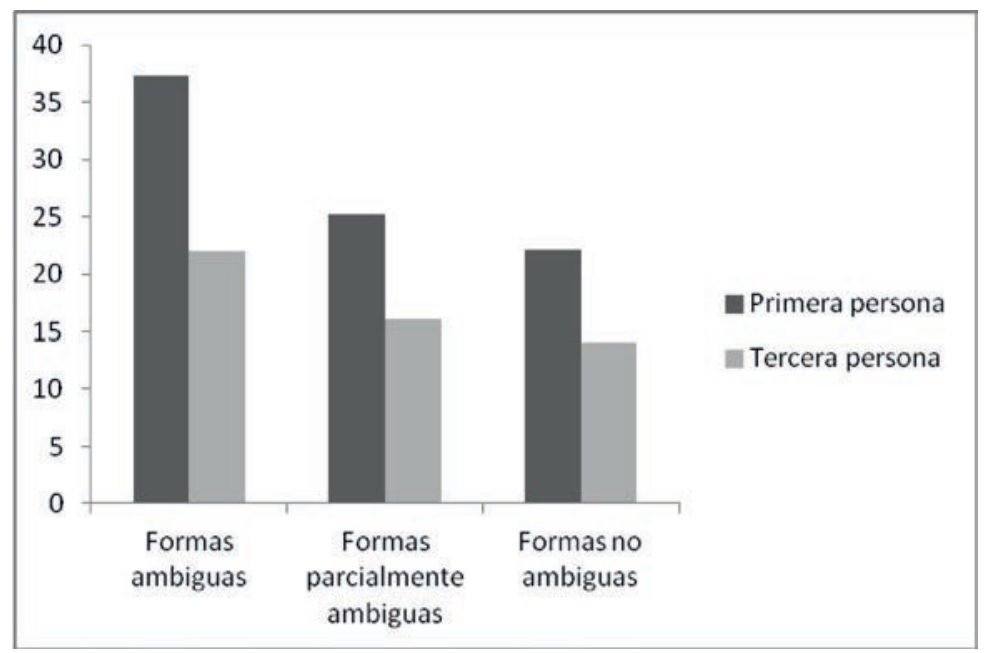

Gráfico 1. Porcentajes de sujeto pronominal expreso según ambigüedad para la primera y tercera personas (prueba de $\chi^{2}$ de Pearson, $\mathrm{p}$-valor $=0.000$ )

Se puede observar que en ambas personas gramaticales, según sean ambiguas o no, se dan diferencias en la aparición del pronombre personal sujeto, aunque es más acusado el aumento para la primera persona del singular cuando es ambigua.

La segunda manera de comprobación puede estar en la consideración de las formas parcialmente ambiguas, como recuerdo en (7), que es una forma verbal que tiene correlato en el ámbito nominal:

(7) Y muy bien/ yo la recuerdo con mucho cariño porque fue un// bueno// hacer una vida en hacer un proyecto de vida juntos// [GRAN-H33-014]

El análisis multivariante, plasmado en la Tabla 3, señala que este tipo de formas también presenta una ligera tendencia a favorecer la expresión del pronombre sujeto.

No podemos dejar de comentar que la tradición, con carácter general, consideró la ambigüedad como un factor poco importante a la hora de explicar la presencia de los pronombres sujeto. Así lo sostiene Enríquez (1984: 119 y ss.). Cita la autora al respecto los trabajos de Ejarque (1977), Rosengren (1974-75) y el suyo propio, entre otros. Aunque no es el caso del trabajo de Enríquez, otros trabajos cuyos corpora se basan en obras literarias nos impiden la comparación. Probablemente, a mayor similitud de corpora, mayor afinidad en los resultados. 


\subsection{SemÁntica VERBAL}

Una de las claves que puede ayudar a la comprensión del funcionamiento del pronombre personal sujeto en nuestro corpus es la semántica verbal. Es este un factor reconocido por los autores como favorecedor de la presencia de este pronombre. Hemos distinguido, siguiendo la guía de codificación de Bentivoglio, Ortiz y Silva-Corvalán (2011), entre verbos que expresan procesos mentales, verbos de estado, verbos dicendi, verbos de actividad y verbos aspectuales. Nosotros añadimos la categoría de verbos de movimiento. Así, dentro de los verbos que expresan procesos mentales, hemos incluido, por ejemplo, comprender, en los verbos de estado, estar; en los verbos dicendi, decir; en los verbos de actividad, jugar; en los verbos aspectuales, acabar; y en los verbos de movimiento, ir.

De esta distinción se deduce que existen verbos que llevan implícito el rasgo de + subjetividad (como los que expresan procesos mentales, del tipo pensar), y otros (como aquellos que indican movimiento, por ejemplo, ir) que carecen de este rasgo. La hipótesis de partida, en este punto, es, pues, que el pronombre personal sujeto tendrá mayor presencia en los verbos del primer grupo frente al resto. Esto queda patente en los resultados de la Tabla 3 , puesto que los verbos de procesos mentales ofrecen un valor de 0,648 , frente al valor menos favorecedor: 0,439 de los verbos de actividad.

En (8) anotamos la expresión del pronombre yo con uno de los verbos, que según Enríquez (1984: 118), son más proclives a llevar de forma explícita el pronombre personal sujeto.

(8) Yo comprendía que estaban// pasándolo mal/ trabajando fuera de su casa/ [GRAN-H32-031].

Enríquez (1984: 118) cita a Rosengren (1974-75: 222) quien destaca específicamente los verbos saber y querer como los que presentan la mayor frecuencia de uso pronominal. Sin embargo, en el corpus que estudiamos, no son esos los verbos privilegiados. Los datos de los principales verbos ${ }^{10}$ de este grupo aparecen en la Tabla 4.

10 Son ocho verbos que suponen 428 de los 591 casos de verbos de este grupo semántico en nuestro corpus. 
Tabla 4. Presencia y ausencia del sujeto pronominal en los principales verbos de "procesos mentales"

\begin{tabular}{|l|c|c|c|c|}
\hline & Presencia & Ausencia & Total & $\%$ de presencia \\
\hline Acordarse & 7 & 30 & 37 & 18,92 \\
\hline Conocer & 3 & 12 & 15 & 20,00 \\
\hline Creer & 47 & 21 & 68 & 69,12 \\
\hline Pensar & 6 & 29 & 35 & 17,14 \\
\hline Querer & 7 & 38 & 45 & 15,56 \\
\hline Recordar & 7 & 26 & 33 & 21,21 \\
\hline Saber & 30 & 82 & 112 & 26,79 \\
\hline Ver & 21 & 61 & 82 & 25,61 \\
\hline
\end{tabular}

Ni qué decir tiene que el dato más llamativo es el porcentaje de presencia del sujeto pronominal con el verbo creer, que muestra un altísimo 69,12\%, seguido de saber $(26,79 \%)$ y ver $(26,79 \%)$, mientras que los demás muestran unos datos más cercanos a la media global de la muestra. Recordemos que la peculiaridad de creer le ha hecho merecedor de tratamiento específico en algún estudio (Aijón y Serrano 2010). Estos autores obtienen un porcentaje de presencia similar al nuestro ( $75 \%$ de los casos), si bien el corpus estudiado es muy diferente (Aijón y Serrano 2010: 13).

A la vista de estos datos, parece que el rasgo [+subjetividad] que está presente en estos verbos no lo explica todo para favorecer la tendencia a la aparición del sujeto, sino que tiene que combinarse con el rasgo [-agentividad], como ocurre con creer, ver y saber frente a querer y pensar, que estarían en el otro extremo de la agentividad.

Además, como todos los factores parecen estar interrelacionados, no podemos dejar de comentar los datos que aparecen si combinamos la variante semántica "proceso mental" con la persona gramatical del verbo. Es lo que hemos procurado hacer en la Tabla 4. 
Tabla 5. Porcentajes de personas gramaticales en los principales verbos de "procesos mentales".

\begin{tabular}{|l|c|c|c|c|c|}
\cline { 2 - 6 } \multicolumn{1}{c|}{} & \multicolumn{5}{c|}{ personas gramaticales } \\
\cline { 2 - 6 } \multicolumn{1}{c|}{} & $1 \mathrm{~s}$ & $2 \mathrm{~s}$ & $3 \mathrm{~s}$ & $1 \mathrm{p}$ & $3 \mathrm{p}$ \\
\hline $\begin{array}{l}\text { \% de presencia en } \\
\text { procesos mentales }\end{array}$ & 85,27 & 11,63 & 2,33 & 0,00 & 0,76 \\
\hline $\begin{array}{l}\% \text { de ausencia en } \\
\text { procesos mentales }\end{array}$ & 69,23 & 15,05 & 3,01 & 8,36 & 4,35 \\
\hline $\begin{array}{l}\text { \% total en procesos } \\
\text { mentales }\end{array}$ & 74,07 & 14,02 & 2,80 & 5,84 & 3,27 \\
\hline
\end{tabular}

Hay varios porcentajes que llaman nuestra atención. El primero es la altísima presencia de la primera persona, con $74,07 \%$ de todos los casos en estos ocho verbos, mientras que para el total de la muestra supone el $24,70 \%$, como se puede comprobar en la Tabla 3. El segundo dato importante es la casi ausencia de la tercera persona del singular, que para estos verbos supone solo el 2,80\% frente al 16,10\% de la totalidad del corpus (Tabla 3). El resto de las personas no presentan oscilaciones significativas. Es cierto que la propia dinámica de la conversación, que pone el foco en la propia experiencia del informante, ayuda a estos datos; pero, no obstante, la diferencia entre los porcentajes de aparición de la tercera persona en la totalidad de la muestra y la aparición de la misma persona con estos verbos, resulta llamativa.

Podemos en este punto anotar la interrelación de factores "semántica" y "persona", puesto que, al estar muy representada la primera persona en este tipo de verbos, la tendencia a la aparición del sujeto pronominal se ve incrementada. Obsérvese cómo hay un $85,27 \%$ de pronombre yo en caso de que aparezca algún sujeto pronominal en este tipo de verbos de "procesos mentales".

Para finalizar este apartado, tenemos que anotar que nuestros datos globales en este punto coinciden con los de otros estudios (Enríquez 1984 y Bentivoglio 1987), mientras que difieren de los obtenidos por Orozco y Guy (2008) y, al menos parcialmente, de los de Aijón y Serrano (2010).

Orozco y Guy (2008) comentan que no encontraron diferencias en un primer momento entre los verbos de actividad mental, los estimativos, los de estado y los de actividad externa -estos empleados, a su vez por Otheguy y Zentella (2012)-.Así pues, redujeron las categorías a dos: verbos de estado, por un lado, y de actividad y estimativos, por otro. Los primeros presentaron el mayor índice de presencia pronominal. Hay que destacar que los propios autores reconocen lo insólito del dato: "La explicación de estos resultados presenta un reto formidable al investigador" (Orozco y Guy 2008: 77). 
Por su parte, Aijón y Serrano (2010: 27) señalan que "Los datos obtenidos confirman que hay diferencias entre hombres y mujeres en la distribución de (yo) creo, y pueden indicar la existencia de estilos comunicativos diferentes". En todo caso, de sus datos parece confirmarse que los hombres usan más el verbo creer, pero no se sigue que haya diferencias generolectales entre el uso de yo сreo / сreo. En nuestro corpus los datos no muestran diferencias en el uso de (yo) creo por parte de hombres y mujeres, puesto que anotamos 67 casos, de los cuales 31 son de hombres y 36 de mujeres. Se ha calculado el estadístico $\chi^{2}$ de Pearson para la tabla de contingencia sexo-expresión del pronombre y el resultado es claramente no significativo $(\mathrm{p}$-valor $=0.538)$.

\subsection{Perífrasis}

Una de las variables que ha resultado significativa en nuestro corpus es la perífrasis verbal en la que distinguimos tres variantes: forma no perifrástica, perífrasis modal y perífrasis aspectual. Como se puede comprobar en la Tabla 3, hay una tendencia a que las formas perifrásticas patrocinen la ausencia del sujeto, frente a las no perifrásticas. Además, se constata que esa tendencia es más acentuada en las perífrasis aspectuales (con 0,367 de peso probabilístico) como en (9), que las modales $(0,467)$, como en (10).

(9) Y yo he dicho/ "pues vamos a montar algo"/ (risas) con las barras de labios nos forramos (risas) [GRAN-H12-021]

(10) O sea yo me puedo fumar un/ un porro/ o meterme lo que quiera siempre y cuando/ a los demás los respete [GRAN-H21-044].

$\mathrm{Al}$ ser la perífrasis un núcleo verbal complejo, podemos pensar en diversos factores que expliquen estos resultados. Puede parecer que el valor aspectual del auxiliar sea determinante, pero hay datos contradictorios que nos obligan a expresarnos con cautela. El primer dato contradictorio es que el aspecto progresivo (como se observa en la Tabla 2, en el que se muestra la ANOVA) no ha resultado significativo y no hay que perder de vista que los núcleos verbales progresivos son perífrasis aspectuales de gerundio del tipo estar + gerundio. Sin embargo, si en las perífrasis aspectuales separamos las perífrasis progresivas de aquellas que no lo son (del tipo ir $a+$ inf.), no obtenemos diferencias apreciables, ya que en las perífrasis aspectuales progresivas el $11,97 \%$ presenta sujeto, mientras que en las no progresivas el sujeto aparece en el 10,12\% de los casos. Además, el peso probabilístico de los verbos aspectuales, como acabar, que es una variante de la variable 
"Semántica verbal", es de 0,487 , que no va pareja con la de las perífrasis aspectuales $(0,367)$-ver Tabla $3-$.

Esto puede hacer pensar en la interrelación con otros factores. Se puede sospechar que la variante "forma perífrástica aspectual" está enmascarada con la semántica verbal, es decir, podríamos intuir que determinadas perífrasis favorecen la aparición de ciertos verbos como auxiliados y que eso sea lo que explique la tendencia de las perífrasis verbales aspectuales a la ausencia más acusada del pronombre personal sujeto. Pero no parece ser así a la vista de la Tabla 6 , en la que hemos cruzado la semántica verbal con los tipos de perífrasis.

Tabla 6. Porcentajes y valores absolutos según la semántica verbal para las perífrasis aspectuales y modales

\begin{tabular}{|l|c|c|c|c|}
\hline Semántica & \multicolumn{2}{|c|}{ Perífrasis aspectuales } & \multicolumn{2}{c|}{ Perífrasis modales } \\
\hline & $\mathrm{n}$ & $\%$ & $\mathrm{n}$ & $\%$ \\
\hline Mental & 37 & 12.98 & 9 & 5,84 \\
\hline Estado & 22 & 7.72 & 23 & 14.94 \\
\hline Dicendi & 18 & 6.32 & 13 & 8,44 \\
\hline Actividad & 167 & 58,60 & 67 & 43,51 \\
\hline Aspectual & 8 & 2,81 & 2 & 1,30 \\
\hline Movimiento & 33 & 11,58 & 40 & 25,98 \\
\hline Total & 285 & 100 & 154 & 100 \\
\hline
\end{tabular}

En efecto, observamos que hay más porcentajes de verbos de procesos mentales (favorecedores de la presencia del sujeto) en las perífrasis aspectuales que en las modales, cuando las perífrasis aspectuales tienen tendencia a la no explicitación del sujeto pronominal. Sí parece congruente la mayor presencia de verbos de actividad en las perífrasis aspectuales $(58,60 \%)$ que en las modales $(43,51 \%)$, ya que los verbos de actividad tienen una ligera tendencia a la menor expresión del sujeto, pero este dato queda anulado por la mayor presencia de verbos de movimiento en las perífrasis modales $(25,98 \%)$ que en las aspectuales $(11,58 \%)$, verbos que muestran tendencias similares a los de actividad.

Queda, para acabar el comentario de las perífrasis, por apuntar a un factor fónico, puesto que se podría plantear la hipótesis de que la longitud de la forma verbal influye en la aparición del pronombre personal sujeto y, como es patente, las formas perifrásticas son más largas que las no perifrásticas. Hemos realizado un recuento grosso modo del número de sílabas de los 
verbos ${ }^{11}$ que presentan sujeto pronominal expreso. Este recuento arroja un promedio de 2,60 sílabas para los verbos no perifrásticos, 5,00 para las perífrasis modales y 5,68 para las perífrasis aspectuales. A falta de un estudio detallado de este factor, en nuestro corpus parece que el mayor número de sílabas influye en la menor presencia del sujeto pronominal.

\subsection{EDAD}

En algunos estudios recientes del fenómeno que nos ocupa, como Orozco y Guy (2008) para Barranquilla (Colombia) y Lastra y Martín (2015) para México DF, ha aparecido un factor social significativo en la ausencia del sujeto pronominal: la edad. Como hemos demostrado en Manjón, Pose y Sánchez (en prensa), los jóvenes muestran cierta tendencia a la no explicitación del pronombre sujeto.

La generación joven utiliza más verbos en segunda persona (239 casos, frente a 126 y 87 de los otros grupos de edad), pero menos pronombre tú expresos, porque hay una tendencia muy acusada de los jóvenes a omitir el sujeto pronominal en el caso de que la segunda persona sea inespecífica ( $8.24 \%$ de presencia del pronombre tú en estos casos, frente a prácticamente el doble en las otras generaciones).

Parece evidente, pues, que los jóvenes se caracterizan por las segundas personas inespecíficas sin pronombre, como las que aparecen en (11):

(11) Ø necesitas pa[ra] esa receta cabello de ángel una lata [de] cabello de ángel/y jamón serrano entonces $\boldsymbol{\emptyset}$ extiendes/ $\boldsymbol{\emptyset}$ untas con el cuchillo/ el/ el cabello de ángel en una de las placas// $\mathrm{y} /$ posteriormente $\boldsymbol{\varnothing}$ vas poniendo lonchas de jamón serrano// encima/// [GRAN-H13-01].

La segunda persona inespecífica constituye un medio de empatizar con el interlocutor, reforzando, en términos de Brown y Gillman (1960), la solidaridad y la familiaridad frente a la cortesía y el poder. Este uso solidario hace que tú en el discurso sustituya al yo y a otros pronombres. Como señala Briz (2001: 85), "el tú es a veces el yo o, de otro modo, el yo implica al tú en lo que dice, o el yo es nosotros o es cualquiera, utilizando morfemas de segunda persona en singular o en plural, pronombres indefinidos, etc.". No podemos

11 Se trata de un recuento provisional basado en el número de sílabas de la escritura, sin tener en cuenta los fenómenos de fonética sintáctica o shandi, que en algunos casos hace que se formen diptongos entre las distintas palabras que forman el núcleo verbal complejo. 
estar seguros de la proyección futura de estas diferencias generacionales, pero sospechamos que puede tener fortuna cuando se constata (Manjón, Pose y Sánchez, en prensa) que son los jóvenes de los niveles educativos altos los que más favorecen la ausencia del pronombre personal sujeto. El Gráfico 2 muestra la diferencia de porcentajes entre los tres niveles educativos considerados. En él se observa la diferencia significativa entre el nivel educativo superior, con una presencia del sujeto de solo el $6.73 \%$, frente a los otros dos niveles, que superan el 15\%.

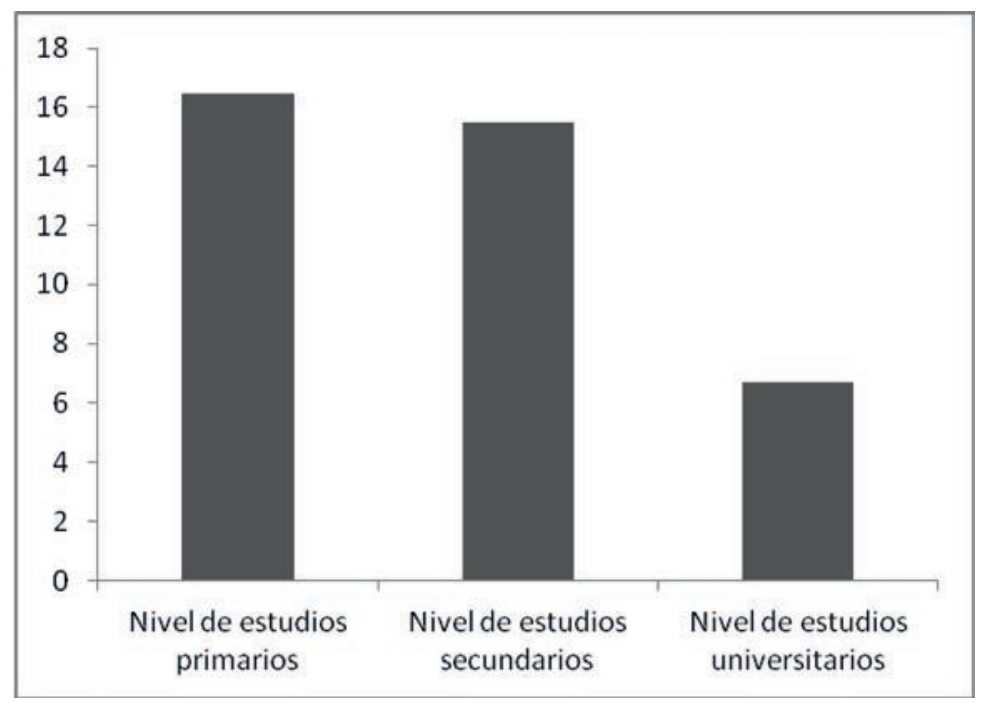

Gráfico 2. Porcentajes de presencia del sujeto pronominal en la primera generación según nivel de estudios (prueba de $\chi^{2}$ de Pearson, $\mathrm{p}$-valor $=0.000$ )

\subsection{CORREFERENCIALIDAD}

La correferencialidad es una variable propuesta como un factor capaz de explicar la presencia / ausencia del pronombre personal sujeto. En nuestro estudio se han considerado las siguientes variantes: correferente con el sujeto previo, como en (12); correferente con otro argumento que no sea sujeto, como en (13); no correferente - casos en los que el sujeto no es mencionado en la oración precedente, como en (14)-; y no aplicable-al comienzo de turno-.

(12) Llegó un momento en/ que vino un señor// de fuera/ de La Línea de la Concepción/// y Ø trajo a sus jugadores/ [GRAN-H31-051] 
(13) Y claro mi abuela siempre ha estado mal/// $\mathrm{mm} /$ la gente iba siempre mucho a/ su casa// a verla y a que pasara el rato porque es que claro/ ella se había dejado mucho// ya no podía ni andar. [GRA H12-019]

(14) De Sevilla pues he visto lo típico/ la Giralda los/// los parques// en fin// la Plaza de España y// lo que sí me gustó mucho fue/ Ø estuvimos en Doñana [GRA-M21-046]

De la tabla 3 se deduce que la no correferencia muestra tendencias neutras en cuanto a la aparición del pronombre, la correferencia con otro argumento patrocina la aparición del pronombre $(0,560)$, mientras que la correferencia con el sujeto previo tiende a inhibir su explicitación $(0,469)$.

Parece que en el discurso hay tendencia a marcar explícitamente el sujeto cuando puede haber riesgo de confusión por el cambio de papel sintáctico del argumento, como en (13) donde 'abuela' pasa de CD de ver-representado por el clítico la-a sujeto de se había dejado-representado por el pronombre ella-; mientras que eso no parece ser necesario cuando el argumento repite el mismo papel sintáctico, como en (12) en el que un señor es sujeto explícito de vino y también es correferente del sujeto omitido de trajo.

\subsection{TURNO}

El último factor que obtuvo valores significativos en nuestro análisis es el del cambio de turno, ya que el inicio de turno muestra un peso probabilístico de 0.581 , es decir, ligeramente patrocinador de la presencia del sujeto pronominal. Es lo que ocurre en (15):

(15) E: Y conectando sus estudios con/ su profesión actual ¿qué puede decir de ella?

I: Pues/ curiosamente que cuando yo estudié la carrera que hice Farmacia nunca pensé que me iba a dedicar a investigación científica como me he dedica[d]o. [GRAN-M33-018]

Como la interrelación de factores es lo usual en el fenómeno que tratamos, es el pronombre personal de primera persona el que tiende a aparecer con el cambio de turno, como ocurre en (15). Queda de manifiesto lo anterior si observamos la Tabla 7 donde aparecen los datos referidos a la presencia y ausencia del sujeto pronominal en los cambios de turno. 
Tabla 7. Presencia y ausencia del sujeto pronominal en los cambios de turno, según persona gramatical

\begin{tabular}{|c|c|c|c|c|}
\hline & Presencia & Ausencia & Total & \% presencia \\
\hline $1^{\text {a }}$ & 69 & 137 & 206 & 33,50 \\
\hline $2^{\text {a }}$ & 3 & 44 & 47 & 6,38 \\
\hline $3^{\text {a }}$ & 2 & 24 & 26 & 7,69 \\
\hline $4^{\text {a }}$ & 4 & 38 & 42 & 9,52 \\
\hline $6^{\text {a }}$ & 1 & 26 & 27 & 3,70 \\
\hline Total & 79 & 269 & 348 & 22,77 \\
\hline
\end{tabular}

Es fácil observar que la mayor parte de la presencia del pronombre corresponde a la primera persona del singular con un 33,50\%, frente a las demás personas que no llegan a alcanzar en ningún caso el $10.00 \%$. Sin duda, las reglas lógicas del juego pregunta-respuesta ayudan a lo anterior.

Para finalizar este subapartado no podemos dejar de comentar que Blas Arroyo (2005) ha señalado que el pronombre de primera persona en el comienzo de turno es una característica discursiva femenina:

Las mujeres - especialmente las de edades más avanzadas- utilizan usos topicalizadores del pronombre de primera persona (yo) al comienzo de un turno de palabra. Un rasgo expresivo que sirve para destacar la participación activa del emisor en el evento que se relata y que podemos considerar un marcador generolectal (Blas Arroyo 2005: 167).

Por el contrario, en el corpus PRESEEA de Granada la presencia de yo al comienzo de turno no está influida por el factor sexo, puesto que de los 69 casos anotados, 34 son de hombres y 35 de mujeres.

\section{CONCLUSIONES}

El análisis y discusión de nuestros datos vienen a corroborar las tendencias halladas en estudios anteriores para otras comunidades hispánicas. Se constata que, en la presencia del sujeto pronominal, están implicados factores como la persona gramatical, su especificidad, la ambigüedad de la forma verbal, su significado, la correferencia y el comienzo del turno de habla. Además, han aparecido algunos factores relativamente novedosos: 
uno es social, como la edad de los hablantes; y otro es lingüístico, como las perífrasis. Esos dos factores, como se observa en la Tabla 3, tienen una fuerza de factor intermedia (138 y 141, respectivamente), entre los que más fuerza tienen, caso de la persona gramatical (fuerza de 446), y los que ocupan los lugares menos prominentes, como el cambio de turno, con una fuerza de 90.

Asimismo, constatamos una tasa pronominal o aparición de los pronombres personales muy baja, con similitudes con comunidades de habla como la del DF mexicano o la de Madrid. Esto aleja al español granadino de las comunidades caribeñas donde la aparición del pronombre llega hasta más del 40\% de los casos (Cameron 1993). Creemos que la explicación tiene que ver con la presencia generalizada de abertura o proyección vocálica que actúa manteniendo la concordancia, del mismo modo que la /-s/ en otras comunidades de habla.

La presencia o ausencia de los pronombres personales sujeto depende de una interrelación de factores difíciles de deslindar. Creemos que hay un factor con la máxima importancia como es la persona gramatical, con dos vertientes. La primera es que la primera persona, el yo, se opone a todas las demás por su alta aparición $(24,7 \%$ y peso probabilístico de 0,646$)$; la segunda consiste en que las personas del singular se oponen a las personas del plural, puesto que estas últimas presentan unos porcentajes de aparición y probabilidades muy pequeños, como se puede comprobar en la Tabla 3.

Este factor clave influye en todos o casi todos los demás, que son de diversa índole. Podemos concluir que la persona interrelaciona con factores morfofonológicos, semánticos y pragmático-textuales.

Los factores que podemos considerar morfofonológicos son la ambigüedad y las formas perifrásticas, especialmente las aspectuales. En lo que toca a la ambigüedad, corroboramos la tendencia hispánica generalizada a usar más el pronombre con las formas equívocas, en especial con los pretéritos imperfectos de indicativo y subjuntivo. Debe tenerse en cuenta que este factor va de la mano de la persona, ya que las formas ambiguas son típicamente las de primera y tercera persona del singular.

A falta de contrastar con otros estudios más detallados, creemos que otro factor morfofonológico lo constituye la longitud de la forma verbal. Esta hipótesis puede explicar que las perífrasis, especialmente las aspectuales, muestren un rechazo significativo a la aparición del pronombre personal sujeto. Claro está que en este punto vuelve a intervenir el factor determinante de la persona verbal, puesto que las formas más cortas del verbo español suelen ser las primeras personas del singular.

Los factores semánticos significativos en el español hablado de Granada han resultado ser la semántica verbal y la especificidad del sujeto. Así, como ocurre en otras comunidades de habla hispánica, los verbos que significan 
procesos mentales del tipo creer, saber o ver, muestran una inequívoca tendencia a su expresión con el sujeto pronominal. Como aparece en la Tabla 6, otra vez es la primera persona del singular la que acapara la inmensa mayoría de las apariciones. Asimismo, debemos recordar que en estos casos se trata de formas verbales cortas, con una o dos sílabas.

La especificidad es el segundo factor semántico que coadyuva a la aparición del sujeto pronominal en español, ya que las expresiones inespecíficas tienen una clara tendencia al rechazo del pronombre personal, puesto que muestran un peso probabilístico de 0,266 -ver Tabla 3-. Además, este factor va de la mano del factor social edad, puesto que son las generaciones más jóvenes las que promueven la inespecificidad en el discurso, como un modo de refuerzo de la solidaridad y familiaridad (Brown y Gillman 1960). Los jóvenes granadinos se caracterizan por el uso abundante de segundas personas inespecíficas sin pronombre.

También existen factores que podemos calificar como pragmáticotextuales que intervienen en las tendencias a la expresión del sujeto pronominal, si bien, en el caso del español hablado en Granada, son los factores que muestran un rango más bajo. Nos referimos a la correferencia y al cambio de turno. En lo que toca a la correferencia, del análisis de nuestros datos se colige que existe una tendencia a explicitar el sujeto pronominal cuando antes ha aparecido desempeñando otra función sintáctica; mientras que la aparición no es tan necesaria cuando el argumento repite el mismo papel sintáctico, es decir, cuando es correferente a la vez semántico y sintáctico.

El cambio de turno promueve la aparición del sujeto pronominal, pero este factor está íntimamente ligado a la persona gramatical, puesto que es absolutamente dominante en esta posición la primera persona del singular, como se ha podido comprobar en la Tabla 7.

Si hacemos una recapitulación final, podemos concluir que, al igual que en otras comunidades de habla, la aparición o ausencia del sujeto pronominal en el español hablado de Granada obedece a múltiples factores. Existe un factor dominante que es la persona gramatical, que creemos que influye de una manera $u$ otra en el resto de factores significativos, que hemos agrupado en morfofonéticos (ambigüedad de la forma verbal, perífrasis verbal), semánticos (semántica verbal, especificidad del sujeto), pragmático-textuales (correferencia, cambio de turno) y sociales (edad). 


\section{REFERENCIAS BIBLIOGRÁFICAS}

Ajón Oliva, Miguel Ángel y María José SerRano. 2010. El hablante y su discurso: expresión y omisión del sujeto de creo. Oralia 13: 7-38.

Barrenechea, Ana María y Alicia Alonso. 1977. Los pronombres personales sujetos en el español hablado en Buenos Aires. En Juan M. Lope Blanch (ed.). Estudios sobre el español hablado en las principales ciudades de América, pp. 333-349. México: UNAM.

Bentivoglio, Paola. 1980. Why canto and not yo canto? The problem of first-person subject pronoun in spoken Venezuelan Spanish. M. A. Thesis, Los Angeles: University of California.

1987. Los sujetos pronominales de primera persona en el habla de Caracas. Caracas: Universidad Central de Venezuela.

Bentivoglio, Paola, Luis A. Ortiz y Carmen Silva-Corvalán 2011. La variable expresión del sujeto pronominal. Guía de codificación. En http://preseea.linguas.net/Portals/0/ Metodologia/guia_codificacion_sujetos_julio_2011.pdf

Blas Arroyo, Jose Luis. 2005. Sociolingüistica del español. Desarrollos y perspectivas en el estudio de la lengua española en contexto social. Madrid: Cátedra.

Briz, ANTonio. 2001. El español coloquial en la conversación. Esbozo de pragmagramática. Barcelona: Ariel Lingüística.

Brown, R.-Gillman, A. 1960. The Pronouns of Power and Solidarity. En Sebeok, TH. (ed.). Style in Language, pp. 252-275. N. York: M.I.T. Press.

CAMERON, RichARD. 1993. Ambiguous agreement, functional compensation, and non-specific tú in the Spanish of San Juan, Puerto Rico and Madrid, Spain. Language Variation and Change 5: 305-334.

Carvalho, Ana M., Rafael Orozco y Naomi Lapidus Shin. 2015. Subject Pronoun Expression in Spanish: A Cross-dialectal perspective. Georgetown University Press.

Claes, Jeroen. 2011. ¿Constituyen las Antillas y el Caribe continental una sola zona dialectal? Datos de la variable expresión del sujeto pronominal en San Juan de Puerto Rico y Barranquilla, Colombia, Spanish in Context 8:2: 191-212.

EJarque, Delia. 1977. El pronombre personal sujeto en Español. Cuadernos de Filología VII: $29-85$.

ENRíQUeZ, EMilia. 1984. El pronombre personal sujeto en la lengua española hablada en Madrid. Madrid: CSIC.

ERKER, DANIEL, \& GREGory R. GuY 2012. The role of lexical frequency in syntactic variability: variable subject personal pronoun expression in Spanish, Language 88: 526-557.

Givón, Talmy. 1976. Topic, pronoun and grammatical agreement. En Charles N. Li (ed.). Subject and topic, pp. 151-188. New York: Academic Press.

Hernández Campoy, Juan Manuel y Manuel Almeida. 2005. Metodología de la investigación sociolingüística. Málaga: Comares.

Hochberg, Judith G. 1986. Functional compensation for /s/ deletion in Puerto Rican Spanish. Language 62: 609-621.

Lastra, Yolanda y Pedro Martín Butragueño. 2015. Subject Pronoun Expression in Oral Mexican Spanish. En Ana M. Carvalho, Rafael Orozco y Naomi Lapidus Shin (eds.). Subject Pronoun Expression in Spanish: A Cross-dialectal perspective, pp. 39-57. Georgetown University Press.

Manjón-Cabeza, Antonio, Francisca Pose y Francisco José Sánchez (en prensa). El factor social edad y la expresión del sujeto pronominal en el español hablado de Granada. Lingüistica Española Actual. 
Moya Corral, Juan Antonio (Coord.). 2007. El Español hablado en Granada. Corpus oral para su estudio sociolingüistico. I Nivel de estudios alto. Univ. de Granada.

(COORD.). 2008. El Español hablado en Granada II. Corpus oral para su estudio sociolingüístico. Nivel de estudios medio. Univ. de Granada.

(COORD.). 2009. El Español hablado en Granada III. Corpus oral para su estudio sociolingüistico. Nivel de estudios bajo. Univ. de Granada.

2013. Rasgos y valoraciones en el oriente de Andalucía. En Antonio Narbona Jiménez (coord.). Conciencia y valoración del habla andaluza. Sevilla: Univ. Internacional de Andalucía.

Orozco, Rafael y Gregory R. Guy. 2008. El uso variable de los pronombres sujetos: ¿qué pasa en la costa Caribe colombiana? En Maurice Westmoreland y Juan Antonio Thomas (eds.). Selected Proceedings of the 4th Workshop on Spanish Sociolinguistics, pp.70-80. Somerville, MA: Cascadilla Proceedings Project.

Otheguy, Ricardo, \& Ana C. Zentella. 2012. Spanish in New York. Language Contact, Dialectal Leveling, and Structural Continuity. Oxford: Oxford University Press.

PeŠKova, Andrea. 2015. Sujetos pronominales en el español porteño. Implicaciones pragmáticas en la interfaz sintáctico-fonológica. Berlin: De Gruyter Mouton.

RAE. Esbozo para una nueva gramática de la lengua española. 1973. Madrid: Espasa-Calpe.

RAE y Asociación de Academias de la Lengua Española. 2009. Nueva Gramática de la Lengua Española. Madrid: Espasa-Calpe.

Ranson, Diana L. 1991. Person marking in the wake of /s/ deletion in Andalusian Spanish, Language variation and Change 3: 133-152.

Rosengren, Per. 1974-75. Presencia o ausencia de los pronombres personales sujeto en español moderno. Estocolmo, Romántica. Gothoburgensia, XIV (Reseña, A. Quilis, RFE. LVII: 326-332).

Sankoff, David, Sali Tagliamonte y ERIC Smith. 2005. GoldVarb X. A variable rule application for Macintosh and Windows. University of Toronto.

Shin, Naomi Lapidus y Ricardo Otheguy. 2013. Social class and gender impacting change in bilingual settings: Spanish subject pronoun use in New York. Language in Society 42: 429-452.

Silva-Corvalán, Carmen. 1982. Subject variation in spoken Mexican-American Spanish. En J. Amastae y L. Elías-Olivares (eds.). Spanish in the United States: Sociolinguistic aspects, pp. 93-120. New York: Cambridge University Press.

2001. Sociolingüistica y pragmática del español. Georgetown Univ. Press.

2003. Otra mirada a la expresión del sujeto como variable sintáctica. En Francisco Moreno Fernández, José Antonio Samper Padilla y otros (eds.). Lengua, variación y contexto : estudios dedicados a Humberto López Morales. Vol. 2, pp. 849-860.

2015. Infantes bilingües y hablantes de herencia adultos: ¿Qué los vincula? Boletín de Filología 50 (1):165-191.

Tejada Giráldez, M. ${ }^{a}$ De la Sierra. 2015. Convergencia y divergencia entre comunidades de habla: a propósito de la /s/ implosiva. Contribución al estudio de los patrones sociolingüísticos del español de Granada. Tesis doctoral. Univ. de Granada. 